\title{
Intrinsic nonlinearity probed by intermodulation distortion microwave measurements on high quality $\mathrm{MgB}_{2}$ thin films
}

\author{
G. Cifariello, M. Aurino, E. Di Gennaro, G. Lamura ${ }^{a}$ and A. Andreone \\ CNR-INFM Coherentia and Department of Physics, \\ University of Naples Federico II, I-80125, Napoli, Italy. \\ P. Orgiani ${ }^{b}$, X. X. Xi \\ Department of Physics, The Pennsylvania State University, \\ University Park, PA 16802, U.S.A. \\ J.-C. Villégier \\ CEA-Grenoble SPSMS/LCP, F38054 Grenoble Cedex 9, France.
}

(Dated: October 8, 2018)

\begin{abstract}
The two tone intermodulation distortion arising in $\mathrm{Mg} B_{2}$ thin films synthesized by hybrid physical-chemical vapour deposition is studied in order to probe the influence of the two bands on the nonlinear response of this superconductor. The measurements are carried out by using a dielectrically loaded copper cavity operating at $7 \mathrm{GHz}$. Microwave data on samples having critical temperatures above $41 \mathrm{~K}$, very low resistivity values, and residual resistivity ratio larger than 10 , are shown. The dependence of the nonlinear surface losses and of the third order intermodulation products on the power feeding the cavity and on the temperature is analyzed. At low power, the signal arising from distortion versus temperature shows the intrinsic $s$-wave behavior expected for this compound. Data are compared with measurements performed on $\mathrm{Nb}$ and $\mathrm{YBa}_{2} \mathrm{Cu}_{3} \mathrm{O}_{7-\delta}$ thin films using the same technique.
\end{abstract}

PACS numbers: $74.70 . \mathrm{Ad}, 74.25 . \mathrm{Nf}$ 
A major factor limiting the performance of passive superconducting devices is the nonlinear response to an applied r.f. field, including the generation of harmonics and IMD products. The nonlinear response of a superconductor has two possible and different origins: extrinsic (like the presence of grains and grain boundaries $\frac{1}{2}$ ) and intrinsic (the nonlinear Meissner effect, NLME $\left.{ }^{2.3}\right)$. The extrinsic nonlinear properties of a superconductor are generally associated to the presence of weak links: the Josephson coupling between grains lowers the first penetration field and increases the level of harmonics and intermodulation distortion (IMD) generation in both $d-\underline{\underline{1}}$ and $s$-wave $\mathrm{e}^{\underline{\underline{\underline{x}}}}$ superconductors. This effect can be safely excluded in epitaxial thin films, where the intrinsic origin of nonlinearity is the only and unavoidable cause degrading the performances of whichever superconducting electronic device.

The intrinsic nonlinear response arises from the backflow of excited quasiparticles at finite temperatures well deep in the Meissner state. Some years ago, Dahm and Scalapino $\frac{5.6}{6}$ showed that at low temperatures and small fields the presence of quasiparticles changes the nonlinear response of a superconductor in a way that is remarkably different depending on the pairing symmetry. In the case of magnesium diboride, they also predicted what would be the influence of the two bands on the IMD behavior ${ }^{\underline{7}}$. More recently, this issue has been analyzed thoroughly by considering also the role of anisotropy and strong coupling effects $\stackrel{8}{*}$. Such theoretical studies give therefore the possibility to probe the gap function by looking at the non linear response of a superconductor in the microwave region. This has been done quite recently by using microstrip resonant techniques on patterned niobium and $\mathrm{YBa}_{2} \mathrm{Cu}_{3} \mathrm{O}_{7-\delta}$ (YBCO) thin films $\frac{9.10}{}$. In this paper we present an experimental study of the NLME in $\mathrm{MgB}_{2}$, using IMD measurements carried out in a dielectically loaded cavity on high quality thin films synthesized by hybrid physical-chemical vapour deposition (HPCVD). We compare the results with data taken on YBCO and $\mathrm{Nb}$ films. Contrary to techniques based on transmission-line resonators, this method probes the microwave properties of unpatterned samples, therefore minimizing the enhancement of current density at the edges, that in principle might rise to extrinsic effects in the electrodynamic response.

$\mathrm{MgB}_{2}$ films were grown on (0001)4H-SiC. The substrate choice is due to its very small $(-0.45 \%)$ lattice mismatch with magnesium diboride. A detailed description of the HPCVD technique has been reported elsewhere 11 12. . X ray diffraction shows that the films grow epitaxially, with the c-axis oriented normal to the substrate and the a-axis parallel to the a-axis of $\mathrm{SiC} \stackrel{11.13}{ }$. These samples show values of $T_{C}$ higher than those reported for bulk 
materials, because of coherent strain induced by the epitaxial growth $\frac{14}{}$. Moreover they are very pure, as indicated by the low residual resistivity (minimum value of $0.26 \mu \Omega \mathrm{cm}$ ).

To investigate the power dependence of the microwave properties of the $\mathrm{MgB}_{2}$ films, we performed in the same system configuration the measurement of the surface losses and of the two-tone intermodulation as a function of the input power at different temperatures below $\mathrm{T}_{c}$. We used an open-ended dielectric single-crystal sapphire puck resonator, similar to the one used in ref. $\stackrel{4}{ }$, excited with a transverse electric mode $\left(\mathrm{TE}_{011}\right)$ and operating at the resonant frequency of $7 \mathrm{GHz}$. The resonator enclosure is made of Oxygen Free High Conductivity (OFHC) Copper, as well as the sample holder, placed in the center of the cavity in close proximity with the dielectric crystal. The sapphire puck $(8 \mathrm{~mm}$ height and $16 \mathrm{~mm}$ diameter) is separated from the copper wall by a thin sapphire spacer (6 mm height and $2.5 \mathrm{~mm}$ diameter). By using a micrometer screw, the puck-to-sample distance can be changed, depending on the material under test, in order to get the maximum sensitivity. The cavity is taken under vacuum using a copper can, which includes a double layer $\mu$ metal shield, and inserted in a liquid helium cryostat. For the measurement of IMD third order products, two closely spaced tones with equal amplitudes at frequencies $f_{1}$ and $f_{2}$ are generated by two phase-locked CW synthesizers. The signals, symmetrically separated around the center frequency of the cavity by an amount $\Delta f$, are combined and applied to the resonant structure. All IMD data presented here are taken with $\Delta f=10 \mathrm{kHz}$, whereas the $3 \mathrm{~dB}$ resonance bandwidth is at least a factor 10 larger at all temperatures below $T_{C}$. The output signals coming from the cavity (the two main tones at $f_{1}$ and $f_{2}$ and the two third-order IMDs at $2 f_{1}-f_{2}$ and $2 f_{2}-f_{1}$ ) are detected using a Spectrum Analyzer. No amplifier is used to avoid unwanted nonlinearities. The small puck-to sample distance (less than $1 \mathrm{~mm}$ ) ensures a very good signal-to-noise ratio even at very low input power level. The first detectable signal from the third order intermodulation products starts at $-140 \mathrm{dBm}$ or less.

We measured four $\mathrm{MgB}_{2}$ films, whose deposition conditions were slightly different, resulting in residual resistivity ratio (RRR) values always greater than 10, and thickness values between 150 and $400 \mathrm{~nm}$. All the samples under test have the same critical temperature $(\sim$ $41 \mathrm{~K}$ ) and a similar behavior as a function of power. For the sake of clarity we will show the experimental results obtained on one sample only, which is the most carefully characterized. This film is $150 \mathrm{~nm}$ thick, with an onset $T_{C}=41.4 \mathrm{~K}, \Delta T_{C}=0.1 \mathrm{~K}$. The surface resistance 
measured at low power levels and at $4 \mathrm{~K}$ is below our instrument sensitivity $(50 \mu \Omega)$.

To probe the intrinsic nonlinear properties of this superconductor, we carefully checked under which conditions the Meissner regime applies, i.e. the applied field is lower than the first penetration field $H^{*}$. To this aim, we have measured the variation of the surface resistance as a function of the applied microwave field at a fixed temperature from $4 \mathrm{~K}$ up to the critical temperature. The average microwave field feeding the cavity has been calculated

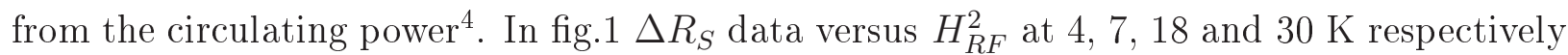
are plotted. The quadratic behavior predicted for $\Delta R_{S}\left(H_{R F}\right)$ in the Meissner state is clearly observed, even if limited to a very small field range at all temperatures. The arrows in the figure indicate the first penetration field $H^{*}$, marking the transition from a quadratic dependence to a linear or sublinear one. The difference in the behavior is confirmed also by measurements of the parameter $\mathrm{r}=\Delta R_{S}\left(H_{R F}\right) / \Delta X_{S}\left(H_{R F}\right) \underline{15}$. For $H_{R F}<H^{*}, \mathrm{r} \ll 1$ (in the range $10^{-2}$ ), as it has to be since in the Meissner regime the nonlinear inductive reactance dominates the nonlinear response. For $H_{R F}>H^{*}, \mathrm{r} \sim 1$, with losses from normal regions driving the electrodynamic behavior of the samples. This has been previously ascribed as due to vortex penetration in grain boundaries ${ }^{4}$. The $H^{*}$ values correspond to circulating powers of 23, 20, 18 and $16 \mathrm{dBm}$ respectively for the temperatures plotted in fig. 1 . Below these power values, the IMD response versus the power circulating inside the cavity $\left(P_{\text {circ }}\right)$ show the expected intrinsic behavior (slope 3) at all temperatures under test. Only at $30 \mathrm{~K}$ data start to depart from a cubic dependence at the highest power levels. In low quality magnesium diboride films grown by dc sputtering, extrinsic effects dominate and the dependence of the IMD products on the r.f. power clearly follows a quadratic law (slope 2$)^{4}$.

In fig. 2 the power $P_{I M D}$ arising from third order products in the $\mathrm{MgB}_{2}$ film at 7 $\mathrm{K}$ is displayed as a function of $P_{\text {circ }}$. The results are compared with similar measurements performed at the same reduced temperature $\left(\mathrm{t}=\mathrm{T} / \mathrm{T}_{C} \sim 0.17\right)$ on a high quality epitaxial $\mathrm{Nb}$ film (200 nm thick) deposited by dc magnetron sputtering ${ }^{16}$ and two highly oriented YBCO films (700 nm thick) grown by thermal evaporation at Theva GmbH. Data are normalized using the factor $P_{N}=10^{-\frac{I L}{20}} Q_{L}$ (IL are the insertion losses expressed in $\mathrm{dB}$ and $Q_{L}$ is the unloaded quality factor) since this allows to remove the dependence of the experimental results on external conditions $\frac{10}{}$. The details of the normalization procedure and of these other experiments will be presented elsewhere.

Following Dahm and Scalapino, the nonlinear response of a superconductor can be de- 
scribed looking at the normalized IMD power because of the relation:

$$
\frac{P_{I M D}}{P_{N}}=K \cdot b^{2}(t) \cdot P_{\text {circ }}^{3}
$$

The quasiparticle backflow contribution of the NLME lies in the nonlinear parameter $b(t)$, that displays a different temperature behavior depending on the gap function symmetry. $K$ is a factor related exclusively to the parameters of the material under test ${ }^{6.8}$. In particular, it strongly depends on the zero temperature London penetration depth $\lambda(0)$, possibly explaining the large difference in the absolute values observed in fig. 2. A similar scattering of data has been previously observed in YBCO films $\frac{17}{}$. Nevertheless, a major feature in the figure is that $\mathrm{MgB}_{2}$, as confirmed also by measurements performed on the other samples, shows a higher level of intrinsic nonlinearities at low temperature and low circulating power in comparison with both $\mathrm{YBCO}$ and $\mathrm{Nb}$.

If we divide eq. 1 by $P_{\text {Circ }}^{3}$, the result becomes independent of the energy level of the e.m. excitation feeding the cavity. The experimentally accessible quantity will depend therefore on $b(t)$ and $K$ only. If we re-scale our experimental data on the theoretical curves ${ }^{5.6 .7 .8}$, we indirectly assume that $K$ has the same $\lambda(0)$-dependence of the theoretical model. In fig. 3 a comparison of the measurements reported here is shown, plotting the nonlinear coefficient $b^{2}$ evaluated at constant circulating powers as a function of temperature for $\mathrm{MgB}_{2}, \mathrm{Nb}$ and YBCO. In the case of the magnesium diboride sample, the dependence is extracted at $P_{\text {Circ }}$ $=0 \mathrm{dBm}$. All experimental data well match the theoretical models 5.6 .7 .8 (solid line, oneband $s$-wave; dash-dotted line, dirty two-band $s$-wave; dotted line, $d$-wave). In the case of YBCO thin films, the nonlinear parameter increases as $1 / T^{2}$ following the prediction for the gap function of a $d$-wave superconductor, and in agreement also with other experiments reported in literature ${ }^{10}$. In $\mathrm{MgB}_{2}$, as well as in $\mathrm{Nb}, b^{2}(t)$ decreases monotonically towards zero, indicating in both cases an $s$-wave behavior. The small residual value at the lowest temperatures is an indication of impurities and/or strong coupling effects ${ }^{8}$. We do not observe any feature in the $b^{2}(t)$ behavior for $\mathrm{MgB}_{2}$ that can be associated with decoupled bands, implying that in high quality samples there is a moderately large value of interband coupling ${ }^{8}$. Moreover, using the analytical formulas reported in ref. ${ }^{7}$, the experimental data seems to match very well the theoretical curve assuming a finite intraband scattering rate $\Gamma$ for both $\sigma$ and $\pi$ bands, in particular yielding a ratio $\Gamma_{\pi} / \Gamma_{\sigma}$ close to 2 .

To conclude, it is worth noting that the peculiarity of $\mathrm{MgB}_{2}$ is that the level of nonlin- 
earity can be decreased changing the scattering rates in the two bands ${ }^{7.8}$. We believe that, playing with substitutional doping, there is still room for improvement, in view of a possible application of magnesium diboride for the development of new passive superconducting devices.

\section{Acknowledgments}

The work at the Department of Physics of the Pennsylvania State University is supported in part by NSF under grant No. DMR-0306746 and by ONR under grant No. N00014-001-0294. The work at University of Naples Federico II is supported in part by PRIN 2004 "Two-gap superconductivity in $\mathrm{MgB}_{2}$ : the role of disorder".

${ }^{a}$ Author to whom correspondence should be addressed. E-mail: gianrico.lamura@na.infn.it.

${ }^{b}$ present address: Dipartimento di Fisica "E. R. Caianiello" and Laboratorio Regionale SuperMat INFM-Salerno, Università degli Studi di Salerno, Baronissi (Sa) I-84081, Italy. 
1 A. T. Findikoglu, P. N. Arendt, S. R. Foltyn, J. R. Groves, Q. X. Jia, E. J. Peterson, L. Bulaevskii, M. P. Maley, and D. W. Reagor, Appl. Phys. Lett. 70, 3293 (1997).

2 S. K. Yip and J. A. Sauls, Phys. Rev. Lett. 69, 2264 (1992).

3 D. Xu, S. K. Yip, and J. A. Sauls, Phys. Rev. B 51, 16233 (1995).

4 G. Lamura, A. J. Purnell, L. F. Cohen, A. Andreone, F. Chiarella, E. D. Gennaro, R. Vaglio, L. Hao, and J. Gallop, Appl. Phys. Lett. 82, 4525 (2003).

5 T. Dahm and D. J. Scalapino, Appl. Phys. Lett. 69, 4248 (1996).

6 T. Dahm and D. J. Scalapino, J. Appl. Phys. 81, 2002 (1997).

7 T. Dahm and D. J. Scalapino, Appl. Phys. Lett. 85, 4436 (2004).

8 E. J. Nicol, J. P. Carbotte, and D. J. Scalapino, cond-mat 0510250 (2005).

9 G. Benz, S. Wünsch, T. A. Scherer, M. Neuhaus, and W. Jutzi, Physica C 356, 122 (2001).

10 D. E. Oates, S.-H. Park, and G. Koren, Phys. Rev. Lett. 93, 197001 (2004).

11 X. H. Zeng, A. V. Pogrebnyakov, A. Kotcharov, J. E. Jones, X. X. Xi, E. M. Lysczek, J. M. Redwing, S. Y. Xu, Q. Li, J. Lettieri, et al., Nature Materials 1, 35 (2002).

12 Z. K. Liu, D. G. Schlom, Q. Li, and X. X. Xi, Appl. Phys. Lett. 78, 3678 (2001).

13 X. H. Zeng, A. V. Pogrebnyakov, M. H. Zhu, J. E. Jones, X. X. Xi, S. Y. Xu, E. Wertz, Q. Li, J. M. Redwing, J. Lettieri, et al., Appl. Phys. Lett. 82, 2097 (2003).

14 A. Pogrebnyakov, J. M. Redwing, S. Raghavan, V. Vaithyanathan, D. Schlom, S. Xu, Q. Li, D. A. Tenne, A. Soukiassian, X. X. Xi, et al., Phys. Rev. Lett. 93, 147006 (2004).

15 M. A. Golosovsky, H. J. Snortland, and M. R. Beasley, Phys. Rev. B 51, 6462 (1995).

16 B. Delaet, P. Feautrier, P. Petmezakis, J.-C. Villégier, A. Benoit, and J.-L. Bret, Nuclear Instruments and Methods in Physics Research A44, 465 (2000).

17 D. E. Oates, S.-H. Park, D. Agassi,and G. Koren, Supercond. Sci. Technol. 17, S290 (2004). 


\section{Figure captions}

Fig. 1: Variation of the surface resistance as a function of the applied microwave field at different temperatures. Arrows set the limit of the quadratic regime.

Fig. 2: The normalized output power of the IMD products as a function of the circulating power for the $\mathrm{MgB}_{2}$ thin film under test $(\triangle)$; an epitaxial $\mathrm{Nb}$ thin film ( $\square$ ); standard high quality YBCO films $(\bullet, \circ)$; at the same reduced temperature $\left(\mathrm{t}=\mathrm{T} / \mathrm{T}_{C} \sim 0.17\right)$. Continuous line illustrates slope 3 .

Fig. 3: The behavior of $b^{2}$ versus the reduced temperature $\mathrm{t}=\mathrm{T} / \mathrm{T}_{C}$. The dotted line represents the theoretical behavior for the $d$-wave case, the solid line for the $s$-wave one-band case, and the dash-dotted line for the $s$-wave two-band case with $\Gamma_{\pi} / \Gamma_{\sigma}=2$; points represent the experimental results for standard high quality YBCO films $(\bullet, \circ)$; an epitaxial $\mathrm{Nb}$ thin film $(\square)$; the $\mathrm{MgB}_{2}$ thin film under test $(\triangle)$ at a circulating power of 0 $\mathrm{dBm}$. 


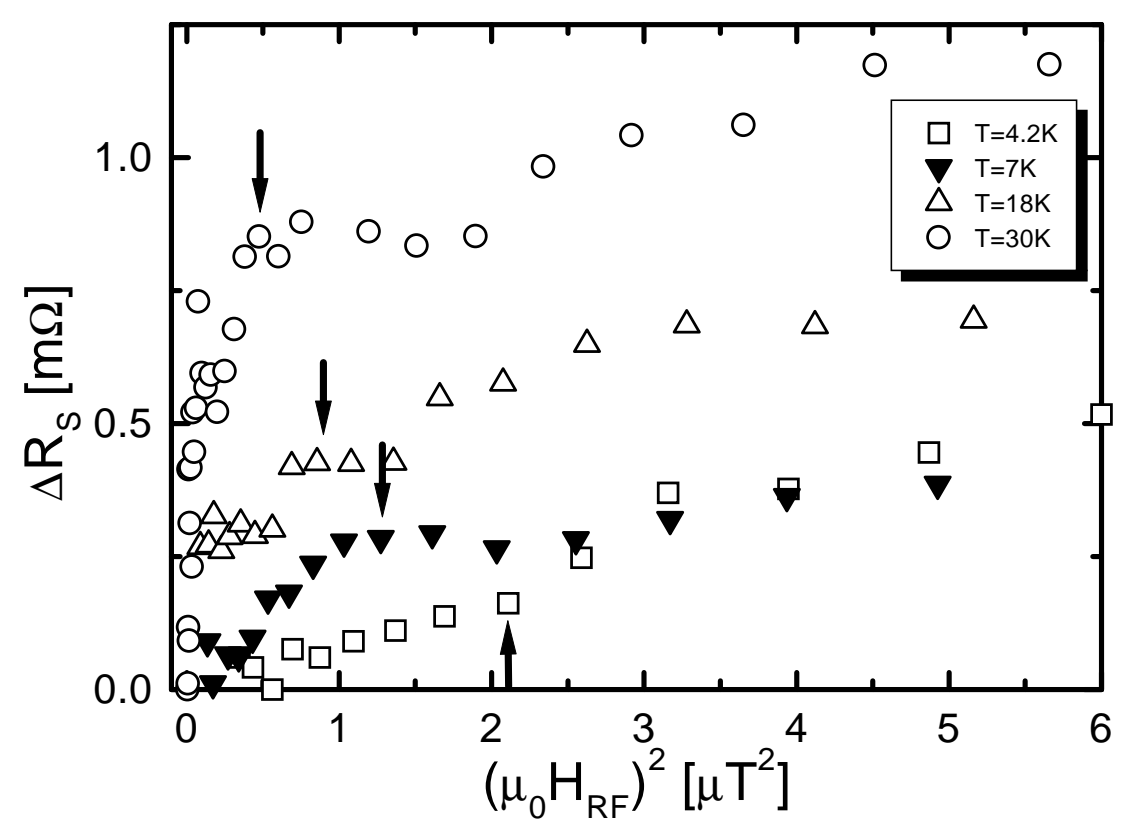




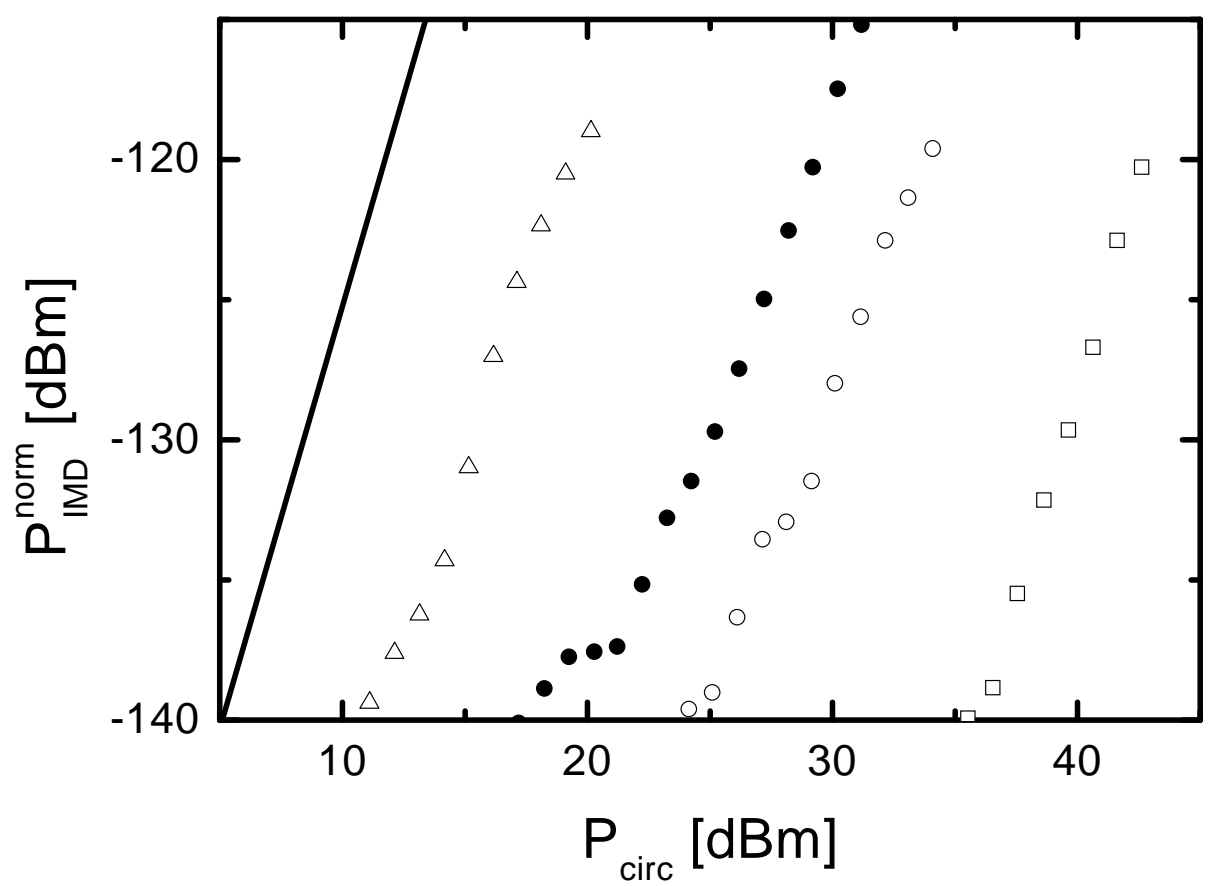




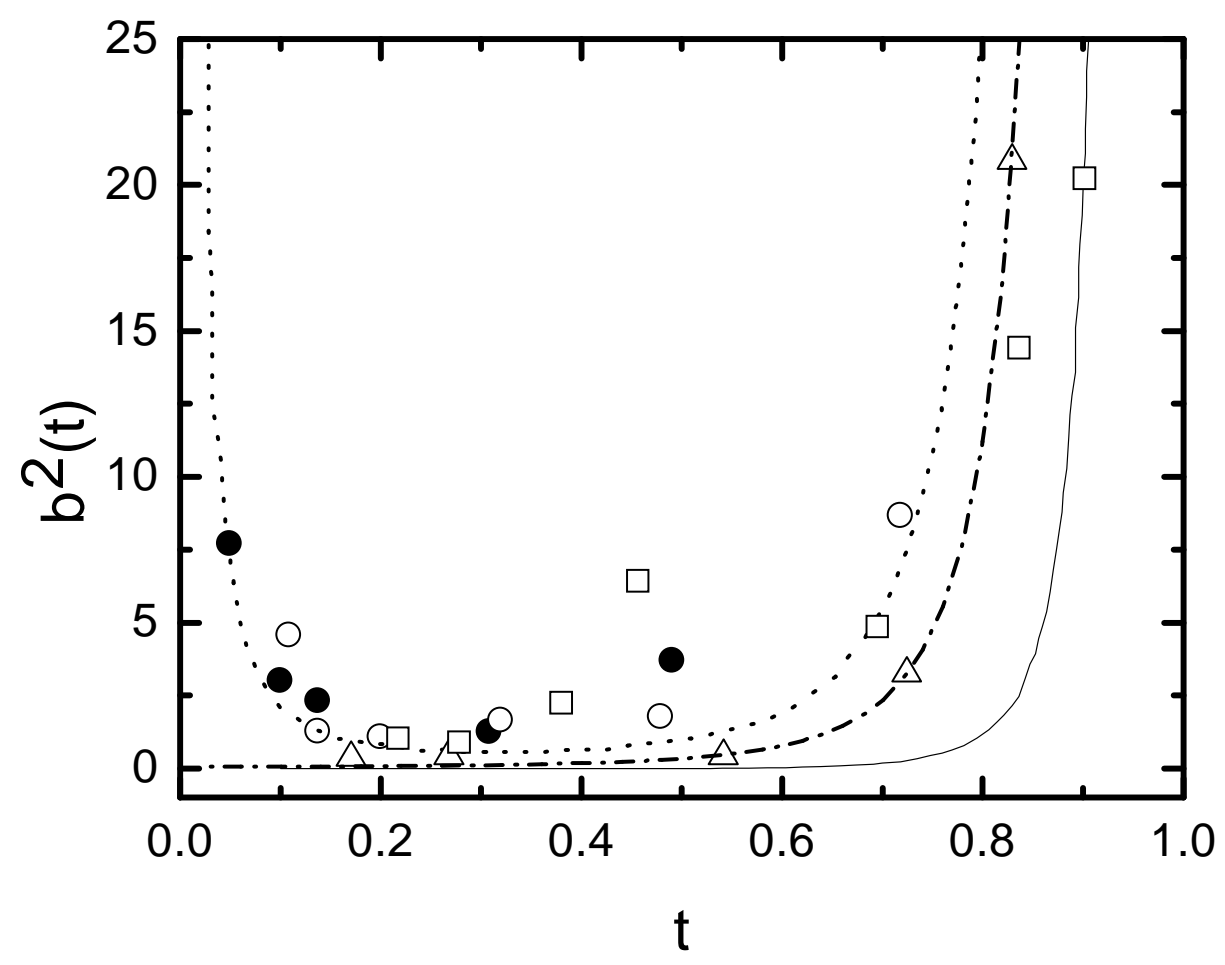

